\title{
Magnetoelectric Macro fiber composite
}

\author{
Ronnie Varghese, ${ }^{1}$ Shree Narayanan, ${ }^{3}$ Donald Leber, ${ }^{3}$ Ravindranath Viswan, ${ }^{2}$ Mingkai $M u,{ }^{3}$ Mohan \\ Sanghadasa ${ }^{4}$ and Shashank Priya ${ }^{1}$ \\ ${ }^{I}$ Center for Energy Harvesting Materials and Systems (CEHMS), Bio- \\ Blacksburg, VA 24061, USA \\ ${ }^{2}$ Materials Science and Engineering Department \\ ${ }^{3}$ Electrical and Computer Engineering Department, \\ Virginia Tech, \\ , USA

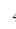 \\ , USA
}

\begin{abstract}
This paper describes the fabrication and performance results of a magnetoelectric macro fiber composite (ME MFC). The magnetoelectric composite was fabricated by bonding a magnetostrictive layer to a piezoelectric layer using a novel approach of low temperature transient liquid phase (LTTLP) bonding. The composite was diced into 150 micron wide fibers and bonded to a custom designed copper flexible circuit using a spin coated low viscosity room temperature curing epoxy. ME MFC's with varying ferrite thicknesses of $0.6 \mathrm{~mm}$ and $0.5 \mathrm{~mm}$ were fabricated and characterized for energy harvesting. The composite with $0.6 \mathrm{~mm}$ ferrite thickness achieved an open circuit voltage of $101 \mathrm{mV}$ (ME voltage coefficient of 6740 $\mathrm{mV} / \mathrm{cmOe}$ ) and peak power of $3.1 \mathrm{nW}$ across $356 \mathrm{k} \Omega$ matching load at $264 \mathrm{~Hz}$.
\end{abstract}

Keywords: macro fiber composite; low temperature bonding; magnetoelectric composite; energy harvesting; magnetic sensor 


\section{Introduction}

Macro fiber composites (MFC) were invented by NASA in 1996 as a surface conformable and flexible piezoelectric ceramic device for controlling structural vibration, noise, and deflections[1]. By being thin, sealed in polyimide and capable of being formed in any geometric shape, such MFC's are versatile enough to be employed as actuators with large deformation and sensors for structural health monitoring and energy harvesting[2]. Therefore, this MFC methodology was chosen to create a flexible magnetoelectric device capable of low frequency AC magnetic field and vibration harvesting. Push-pull type ME composite laminates have previously been found to be extremely sensitive magnetic sensors with low noise[3]. In such sensors, the magnetostrictive layers are bonded externally to laminated piezoelectric fibers. Our macro fiber composites employed magnetoelectric composite fibers in which the magnetostrictive layer was directly bonded internally to the piezoelectric layer.

Magnetoelectric (ME) composites have been found promising for sensors, phase shifters, filters, and tunable transformers. Such devices usually consist of a composite structure that includes a magnetostrictive phase in contact with a piezoelectric phase. The efficiency of the elastic coupling between the two phases depends upon the strain transfer occurring at the interface. Magnetoelectric response of a ME composite is measured as ME voltage coefficient $\alpha_{\mathrm{ME}}$ and the relationship between the magnetic field induced strain in the magnetostrictive material and the generated electric field in the piezoelectric material is given by[4]:

$$
\alpha_{M E}=\left|\frac{\partial T}{\partial S} \times \frac{\partial D}{\partial T} \times \frac{\partial E}{\partial D}\right| \times \frac{\partial S}{\partial H}
$$


where $\mathrm{S}$ is the mechanical strain, $\mathrm{T}$ is the mechanical stress, $\mathrm{D}$ is the electric displacement, $\mathrm{E}$ is the electric field, and $\mathrm{H}$ is the magnetic field.

The magnetoelectric response can be maximized by improving the interfacial properties in terms of matching the mechanical impedance between the magnetostrictive and piezoelectric layers $[5,6]$. Epoxy bonding has been found to perform better than both a Ag-Si alloy with $600^{\circ} \mathrm{C}$ working temperature and a thin borosilicate with $500-600^{\circ} \mathrm{C}$ bonding layers[7]. ME laminate composites are mostly fabricated by bonding a magnetostrictive material to a piezoelectric macro fiber composite [8] or a piezoelectric laminate composite[9]. The mechanical coupling is poor due to the polymer laminate between the piezoelectric MFC and the magnetostrictive layer. In ME thin film composites, the addition of a Pt layer between the piezoelectric film and the magnetostrictive film has been shown to double the magnitude of ME coefficient [10]. In the case of bulk ME composites, the addition of an embedded metallic layer has also resulted in improved ME performance by suppressing interdiffusion between the cofired piezoelectric and magnetostrictive layers[11]. These co-fired ME composites limit the choice of the magnetostrictive, conductive and piezoelectric materials to that which can withstand $>800^{\circ} \mathrm{C}$ processing $[11,12]$. Transient liquid phase (TLP) bonding has been in use for centuries but has recently come to prominence in aerospace and semiconductor industries for the joining of two metallic surfaces[13]. The process entails a thin interlayer metal containing a melting point depressant that melts and fills the voids of the two metal surfaces in contact. This depressant metal diffuses into the parent metal, undergoes isothermal solidification and then upon cooling, the joint becomes homogeneous. Another variant of TLP bonding that employs a low temperature melting solder is low temperature transient liquid phase bonding (LTTLP)[14]. In this latter case, ceramic or metal surfaces are bonded by utilizing a base metal coating on each of 
the mating surfaces and then adding a low melting solder between the base metal layers on each bonding surface. At the solder melting point, some of the base metal dissolves in the solder and undergoes isothermal solidification. The resultant solder-base metal alloy has a higher eutectic point than the solder melting point and therefore, the resultant bond can withstand higher temperatures than the temperature at which bonding occurred.

Building upon these prior findings, we demonstrate here a metallic bonding process for attaching the piezoelectric and magnetostrictive layer in ME laminate composites. The constraints on the bonding process included the thin dimensions of the interface and process temperature below the Curie temperature of the magnetostrictive and piezoelectric layers. Using this low temperature metallic bonding technique, the ME composite was fabricated and then singulated into fibers. These fibers were then bonded to a flexible electrical circuit to realize the first magnetoelectric macro fiber composite (to our knowledge at time of publication).

\section{Experimental Procedures}

The ceramic raw materials were acquired from Piezo Systems (5A4E $0.127 \mathrm{~mm}$ sheet) and Electroscience (Type 40011 ferrite tape). The nickel coating of the piezo sheets were wet etched off using TFG nickel etchant from Transene at $60^{\circ} \mathrm{C}$. After that they were cut into $21 \mathrm{~mm}$ squares. The ferrite tape was fired as recommended by Electroscience (Fig 1 (a)). Indalloy 1E (In 52\%, Sn $48 \%$ ) with eutectic point $118^{\circ} \mathrm{C}$ was chosen for the bonding due to the ease of availability and cost. Both ceramics were coated with $1000 \AA$ Au over a $1000 \AA$ Ti adhesion layer as Au forms a high eutectic point interlayer with In and $\mathrm{Sn}\left(\mathrm{Au}-\mathrm{In}\right.$ eutectic point occurs at $465^{\circ} \mathrm{C}$ whilst that for Au-Sn occurs at $280^{\circ} \mathrm{C}$ ). Solder alloy with $10 \mathrm{~m}$ was deposited using a high throughput 
ultrasonic jet vapor deposition process (Jet Process Corporation, North Haven, CT). These single side coated ferrite and piezo sheets were then pressed down with a $100 \mathrm{gm}$ weight at $125^{\circ} \mathrm{C}$ for an hour in the forming gas environment of an alloying station.

The ME composite was then coated with room temperature silver paste on top of the piezo (Fig 1 (b)) and tested for $\mathrm{d}_{31}$ mode ME performance. No poling was required as we used the piezo in vendor poled $\mathrm{d}_{31}$ mode. Next the Ag paste was removed in acetone and Pt was deposited through a shadow mask with $200 \mathrm{~m}$ line width and $500 \mathrm{~m}$ spacing (Fig 1 (c)). The piezo was repoled at $4000 \mathrm{~V} / \mathrm{mm}$ (which is within the Piezo Systems' recommended 50-100 V/mil) condition. The Pt IDE coated ME composite was then tested for $\mathrm{d}_{33}$ mode ME performance. After testing, the $\mathrm{Pt}$ was polished off using a fine grit sand paper.

The MFC fabrication process is depicted in Fig. 2. The ME composite was diced into $150 \mathrm{~m}$ wide fibers (Fig 3(a)) with a 3mil resin blade with $45 \mathrm{~m}$ diamond size particles (Semicon Tools Inc). For the MFC's flexible circuit, an interdigitated electrode (IDE) pattern of $150 \mathrm{~m}$ line width copper interconnects spaced at $150 \mathrm{~m}$ was designed and printed (Fig 3(b)). Moog Inc. (Galax, VA) made the flexible circuits from Dupont Pyralux LF8510 tape and for ease of wire bonding provided immersion Ag finish on the contact pads.

The flexible circuit was spin coated with Extreme 2115 epoxy of viscosity $250 \mathrm{cP}$ and vacuum bonded for 12 hours on one side of diced ME composite fibers at room temperature. The MFC was poled at Piezo Systems' recommended $4000 \mathrm{~V} / \mathrm{mm}$ condition. The finished MFC is shown in Fig 3 (c) and a SEM cross section of the MFC is shown in Fig 3 (d). 
The MFC was clamped in fixed-free or cantilever mode using a non-magnetic polycarbonate clamp mounted onto a TMC Solution TJ-2 electromagnetic shaker (Fig.4). The shaker was powered by an HP 6825A power supply/amplifier operating as a fixed gain amplifier. The mechanical resonance of each MFC was determined from the velocity frequency response function (FRF) between the tip of the MFC and the reference acceleration measured at the clamp using an accelerometer (Piezotronics Inc. model \# U352C67). The output signal from the accelerometer was conditioned using a charge amplifier (Piezotronics Inc.). The velocity at the tip of cantilever beam was measured using a digital vibrometer (Polytec OFV 353). Polytec 5000 controller was used to generate input signals to the seismic shaker to create vibration and also to capture the output signals from accelerometer and vibrometer. A translatable stage was used to pair a magnet with another in the clamp to create a DC magnetic bias parallel to the length of the MFC (see Fig. 4). The AC magnetic field was generated by a Helmholtz coil of dimension 34 mm width $\times 57 \mathrm{~mm}$ diameter with $28 \Omega$ coil resistance and applied parallel to the DC bias. The voltage FRF of each MFC was obtained by varying the frequency of the AC magnetic field at values close to the first bending resonance obtained from the velocity FRF. The first bending resonance was chosen as it has the highest ME coupling efficiency[15]. SigLab 20-42 data acquisition system with four input channels and two output channels was used for the voltage measurements. This data acquisition system has an internal resistance of $995 \mathrm{k} \Omega$ and this resistance was in parallel with any load resistance attached externally to the device under the test. Voltage generated by the harvester and the power loading curves were generated by placing a digital IET Labs load resistor in series with the harvesters.

\section{Results and Discussion}


As shown in the inset of Fig. 5(a) and (b), the ferrite 40011 has a low coercive field of the order of $\sim 20$ Oe (saturation magnetization $72.4 \mathrm{emu} / \mathrm{g}$ ) and a saturation magnetostriction of $12 \mathrm{ppm}$ at 1767 Oe respectively.

The resonant frequency and ME voltage coefficients for the ferrite ME composites operating in $\mathrm{d}_{31}$ and $\mathrm{d}_{33}$ modes is shown in Fig. 6 and listed in Table 1. The applied AC magnetic field was 1 Oe at $1 \mathrm{kHz}$ with a saturation DC magnetic bias of 21.9 Oe and 27.8 Oe respectively for the 0.5 $\mathrm{mm}$ and $0.6 \mathrm{~mm}$ ferrite $\mathrm{ME}$ composites. In trilayer symmetric ME laminates, the first longitudinal frequency is proportional to the inverse of its length whilst for bilayer asymmetric ME laminates like an ME unimorph, the first bending resonance is lower than that of the longitudinal and proportional to the inverse of the square of the composite's length[15]'[9]. This explains the low resonance frequencies of the $d_{31}$ and $d_{33}$ ME unimorphs prior to their singulation into ME fibers. The ME voltage coefficients are shifted to the left on the $\mathrm{d}_{33}$ mode vs. the $\mathrm{d}_{31}$ mode as the $\mathrm{d}_{33}$ mode electrode pattern (Fig 1 (c)) is electrically unidirectional whilst the $\mathrm{d}_{31}$ mode pattern is bidirectional (Fig 1 (b)). In addition, the $2^{\text {nd }}$ resonance was obtained too close to the $1^{\text {st }}$ than expected of such a bilayer[15]. Unlike the piezo, which were diced into $21 \mathrm{~mm}$ squares, the square cut ferrite LTCC tapes on sintering formed into non-square, slightly rectangular profiles. For an unclamped rectangular plate, the frequency ratio between the $2^{\text {nd }}$ and $1^{\text {st }}$ modes for length:width ratios of $1,1.5$ and 2.5 are 1.47, 1.07 and 1.53 [16]. From Table 1, the ratio between modes for our slightly rectangular ME unimorph plates $(1<$ length:width ratio $<$ 1.5) range between 1.26-1.63 and thus mirroring the performance of an unclamped rectangular plate with length:width ratios between 1 and 1.5 . For comparison, the $2^{\text {nd }}$ to $1^{\text {st }}$ mode frequency ratio for a clamped rectangular cantilever for length:width ratios of 1, 1.5 and 2.5 are 2.44, 3.56 and 5.21 [16]. For further confirmation, we cut a piezo sheet to the same size and shape of the 
ferrite (i.e. slightly rectangular) and conducted a frequency impedance analysis. The $1^{\text {st }}$ resonance was at $59.6 \mathrm{kHz}$ with a $2^{\text {nd }}$ mode at $87.6 \mathrm{kHz}$ and $80.6 \mathrm{kHz}$ with a $2^{\text {nd }}$ mode at 97.8 $\mathrm{kHz}$ for $\mathrm{d}_{33}$ and $\mathrm{d}_{31}$ modes respectively - a mode ratio of 1.47 and 1.21 respectively. Therefore, the $2^{\text {nd }}$ peak is due to the $2^{\text {nd }}$ resonance mode of an unclamped 'slightly rectangular' plate. A perfectly square resonator is known to have a whispering gallery effect - i.e. a single high quality factor resonance due to combination of both $\mathrm{x}$ and $\mathrm{y}$ lateral modes[17].

It has been reported that the L-L mode (longitudinal magnetized-longitudinal poled) has the largest ME voltage coefficient of all the four basic modes namely longitudinal-longitudinal (LL), transverse-longitudinal (T-L), L-T and T-T [9]. This is usually assumed to be due to the higher longitudinal piezoelectric coefficient $d_{33}$ over the transverse coefficient $d_{31}$. In case of a push-pull type configuration where the ME composite consists of numerous ME elements with adjacent piezo elements poled in opposite directions, the ME voltage coefficient was found to be better due to the higher elemental capacitance[9]. Despite the fact that this higher capacitance is in parallel across the IDE pairs, the total device capacitance is extremely low for a fiber based piezoelectric device and causes the required matching load impedance to be elevated.

From the velocity FRF's between the tip of the MFC and the reference acceleration, the $1^{\text {st }}, 2^{\text {nd }}$ and $3^{\text {rd }}$ bending modes and the longitudinal mode of the magneto mechanical resonance for the $0.5 \mathrm{~mm}$ ferrite based MFC was determined to be $259 \mathrm{~Hz}, 594 \mathrm{~Hz}, 1107 \mathrm{~Hz}$ and $1172 \mathrm{~Hz}$ (Figure 7). The voltage FRF does confirm all the modes except for the $3^{\text {rd }}$ bending mode which was insignificant in value. The FRF's of each MFC was obtained by varying the frequency of the AC magnetic field. 
As shown in Figure 8 and summarized in Table 2, two ME MFC's, with ferrite thicknesses of $0.6 \mathrm{~mm}$ and $0.5 \mathrm{~mm}$, operating in $\mathrm{d}_{33}$.mode achieved open circuit voltage of $101.1 \mathrm{mV}$ and 93.8 $\mathrm{mV}$ (ME voltage coefficient of $6740 \mathrm{mV} / \mathrm{cmOe}$ and $6256 \mathrm{mV} / \mathrm{cmOe}$ ) under an AC magnetic field of 1 Oe. The peak power was found to be $2 \mathrm{nW}$ across $555 \mathrm{k} \Omega$ and $1.7 \mathrm{nW}$ across $510 \mathrm{k} \Omega$ matching resistor load. The power measurements were conducted at the first bending resonances of $264 \mathrm{~Hz}$ and $259 \mathrm{~Hz}$ AC magnetic field of $1 \mathrm{Oe}$ (the vibrational force was $\sim 0.01 \mathrm{~g}$ ) with applied DC magnetic bias of 22 Oe and 28 Oe respectively. As an AC magnetic field sensor, the sensitivity of these MFC's of ferrite thicknesses of $0.6 \mathrm{~mm}$ and $0.5 \mathrm{~mm}$ were $1011 \mathrm{mV} / \mathrm{mT}$ and $938 \mathrm{mV} / \mathrm{mT}$ respectively - similar in capability to that of commercially available Hall effect sensors[18].

Due to the $995 \mathrm{k} \Omega$ resistance of the data acquisition system (which is in parallel to the $555 \mathrm{k} \Omega$ and $510 \mathrm{k} \Omega$ matching resistor loads for the $0.6 \mathrm{~mm}$ and $0.5 \mathrm{~mm}$ ferrite ME MFC's), the effective load seen by the transducer were $356 \mathrm{k} \Omega$ and $337 \mathrm{k} \Omega$ for the two ME MFC's of ferrite thickness $0.6 \mathrm{~mm}$ and $0.5 \mathrm{~mm}$ respectively. Therefore, the peak power can be recomputed to be $3.1 \mathrm{nW}$ across $356 \mathrm{k} \Omega$ and $2.6 \mathrm{nW}$ across $337 \mathrm{k} \Omega$ load for the ME MFC of ferrite thickness $0.6 \mathrm{~mm}$ and $0.5 \mathrm{~mm}$ respectively. This matching impedance amounts to an effective electrical capacitance of $1.69 \mathrm{nF}$ and $1.82 \mathrm{nF}$, respectively. After dicing, MFC fabrication and poling, the measured capacitance was $0.14 \mathrm{nF}$ and $0.118 \mathrm{nF}$ at $1 \mathrm{kHz}$ respectively. The discrepancy between the capacitance calculated from the matching impedance and that measured prior to poling is due to a) the frequency difference between the capacitance measurement $(1 \mathrm{kHz})$ and that of the operation (264 and $259 \mathrm{~Hz}$ ) and b) resonant operation of the magnetically induced vibration of the piezoelectric. 
The power generated by these MFC's can be improved by lowering the impedance - i.e. by increasing the capacitance of these fibers. In a MFC, the IDE capacitor pairs across each piezo fiber are in parallel and the individual capacitances add up. As shown in equation (2), the capacitance between each IDE pair of electrodes can be increased by decreasing the distance ' $d$ ' between the copper IDE electrodes, increasing the width ' $w$ ' and the length ' 1 ' of the electrodes [19]:

$$
C=\frac{2 \varepsilon_{r} \varepsilon_{0} l}{\pi} \ln \left[(1+w / 0.5 d)+\sqrt{(1+w / 0.5 d)^{2}-1}\right]
$$

Increase in length of the each IDE capacitor pair is indirectly realized by increasing the breadth of the piezoelectric fibers (can increase stiffness of the whole MFC). However, increasing electrode width decreases the active area of the MFC and also increases the 'dead zone' underneath the electrodes[20]. Nonetheless, similar to push-pull ME composite laminates, higher capacitance MFC's are the best for voltage generation applications[21].

To lower the resonance frequency of these MFC's, whilst maintaining the same dimensions, one can a) add an inertial tip mass - use of an active (i.e. magnetic) tip mass has been shown to improve ME performance and lower resonance[22], b) decrease stiffness of the support and c) decrease stiffness of the Magnetoelectric composite. The stiffness of the support, the Copper IDE kapton flexible circuit, can be adjusted by using a tape with less copper (less copper thickness, more space between copper lines, etc.), thinner kapton tape and finally, with a low modulus of elasticity epoxy between the copper. The stiffness of the ME composite can be adjusted by using large kerf widths (or conversely, smaller piezo fiber breadth) between the ME composite fibers and again by utilizing a low modulus of elasticity epoxy between the fibers. 
Furthermore, ME voltage can be improved by creating ME MFC's using highly magnetostrictive material like Terfenol-D, Metglas, NCZF, etc $[6,8,9,11]$ with highly piezoelectric material like PMN-PT[12]. In addition, ME MFC's that utilize self-biased ME composite materials [23]will expand the capability to applications where a DC bias field cannot be applied.

\section{Conclusion}

We developed a low temperature jet vapor solder bonded $\left(<125{ }^{\circ} \mathrm{C}\right)$ magnetoelectric composite fibers and incorporated them with kapton based copper flexible circuit using a room temperature curing epoxy. With this bonding approach, we achieved an ME voltage coefficient of 6740 $\mathrm{mV} / \mathrm{cmOe}$ and $6256 \mathrm{mV} / \mathrm{cmOe}$ at $264 \mathrm{~Hz}$ and $259 \mathrm{~Hz}$ for $0.6 \mathrm{~mm}$ and $0.5 \mathrm{~mm}$ ferrite based MFC's respectively. The resulting magnetoelectric macro fiber composites provided both low frequency vibration and magnetic energy harvesting and $\mathrm{AC}$ magnetic field sensing capabilities. As an $\mathrm{AC}$ magnetic field sensor, the sensitivity of these MFC's was $1011 \mathrm{mV} / \mathrm{mT}$ and $938 \mathrm{mV} / \mathrm{mT}$ respectively for the two ferrite thicknesses of $0.6 \mathrm{~mm}$ and $0.5 \mathrm{~mm}$. We also explain the various approaches to optimize the power generation capability of these magnetoelectric macro fiber composites. In this paper, we thus report simultaneously the use of a new bonding technique for magnetoelectric composites and the fabrication and operation of the first magnetoelectric macro fiber composite. Such flexible ME MFC's can be utilized as magnetic field actuators and sensors over curved surfaces.

Acknowledgements: The authors gratefully acknowledge the financial support from Air Force Office of Scientific Research (AFOSR) through Young Investigator Program and Office of Basic Energy Science, Department of Energy (S.P.). We are also greatly indebted to Justin 
Farmer CEHMS Laboratory manager for help with the experimental setup. We also would like to thank Bret Halpern of Jet Process Corporation, North Haven, CT for the solder deposition and T.J. Belton and Travis Belton of Moog Components Group Galax Operations, Galax, VA for flexible circuit fabrication.

\section{References}

[1] J.W. High, W.K. Wilkie, Method of fabricating NASA-standard macro-fiber composite piezoelectric actuators: National Aeronautics and Space Administration, Langley Research Center; 2003.

[2] H.A. Sodano, G. Park, D.J. Inman, An investigation into the performance of macro-fiber composites for sensing and structural vibration applications, Mechanical Systems and Signal Processing, 18(2004) 683-97.

[3] D. Shuxiang, J.-F. Li, D. Viehland, Ultrahigh magnetic field sensitivity in laminates of TERFENOL-D and $\mathrm{Pb}(\mathrm{Mg} 1 / 3 \mathrm{Nb} 2 / 3) \mathrm{O} 3-\mathrm{PbTiO} 3$ crystals Applied Physics Letters, 83(2003) 2265-7.

[4] K.-H. Cho, S. Priya, Direct and converse effect in magnetoelectric laminate composites, Applied Physics Letters, 98(2011) 232904 - -3.

[5] C.-W. Nan, G. Liu, Y. Lin, Influence of interfacial bonding on giant magnetoelectric response of multiferroic laminated composites of $\mathrm{Tb}$ [sub $1-\mathrm{x}] \mathrm{Dy}$ [sub x] Fe[sub 2] and $\mathrm{PbZr}[\mathrm{sub}$ x]Ti[sub 1 - x]O[sub 3], Applied physics letters, 83(2003) 4366-8.

[6] G. Liu, C.-W. Nan, N. Cai, Y. Lin, Dependence of giant magnetoelectric effect on interfacial bonding for multiferroic laminated composites of rare-earth-iron alloys and lead--zirconate-titanate, Journal of Applied Physics, 95(2004) 2660-4.

[7] V. Gheevarughese, U. Laletsin, V. Petrov, G. Srinivasan, N. Fedotov, Low-frequency and resonance magnetoelectric effects in lead zirconate titanate and single-crystal nickel zinc ferrite bilayers, Journal of Materials Research, 22(2007) 2130-5.

[8] T. Lafont, L. Gimeno, J. Delamare, G. Lebedev, D. Zakharov, B. Viala, et al., Magnetostrictive-piezoelectric composite structures for energy harvesting, Journal of Micromechanics and Microengineering, 22(2012) 094009.

[9] J. Zhai, Z. Xing, S. Dong, J. Li, D. Viehland, Magnetoelectric Laminate Composites: An Overview, Journal of the American Ceramic Society, 91(2008) 351-8.

[10] P. Zhao, Z. Zhao, D. Hunter, R. Suchoski, C. Gao, S. Mathews, et al., Fabrication and characterization of all-thin-film magnetoelectric sensors, Applied physics letters, 94(2009) 243507-3.

[11] C.-S. Park, S. Priya, Cofired Magnetoelectric Laminate Composites, Journal of the American Ceramic Society, 94(2011) 1087-95.

[12] Y. Yan, Y. Zhou, S. Priya, Giant self-biased magnetoelectric coupling in co-fired textured layered composites, Applied Physics Letters, 102(2013) 052907.

[13] W.D. MacDonald, T.W. Eagar, Transient Liquid Phase Bonding, Annual Review of Materials Science, 22(1992) 23-46. 
[14] J.W. Roman, T.W. Eagar, Low stress die attach by low temperature transient liquid phase bonding, The International Society for Hybrid Microelectronics (ISHM) Symposium Proceedings 1992, pp. 1-6.

[15] J.G. Wan, Z.Y. Li, Y. Wang, M. Zeng, G.H. Wang, J.-M. Liu, Strong flexural resonant magnetoelectric effect in Terfenol- $\mathrm{D} /$ epoxy- $\mathrm{Pb}(\mathrm{Zr}, \mathrm{Ti}) \mathrm{O}$ [sub 3] bilayer, Applied Physics Letters, 86(2005) 202504.

[16] R.D. Blevins, Formulas for natural frequency and mode shape, New York: Van Nostrand Reinhold Co.; 1979.

[17] G. Wei-Hua, H. Yong-Zhen, L. Qiao-Yin, Y. Li-Juan, Whispering-gallery-like modes in square resonators, Quantum Electronics, IEEE Journal of, 39(2003) 1106-10.

[18] http://www.gmw.com/magnetic sensors/asahi/linear-hall-effect-ic.html.

[19] J.Z. Chen, A.A. Darhuber, S.M. Troian, S. Wagner, Capacitive sensing of droplets for microfluidic devices based on thermocapillary actuation, Lab on a Chip, 4(2004) 473-80.

[20] C.R. Bowen, A. Bowles, S. Drake, N. Johnson, S. Mahon, Fabrication and finite element modelling of interdigitated electrodes, Ferroelectrics, 228(1999) 257-69.

[21] J.M. Lloyd, Electrical properties of macro-fiber composite actuators and sensors: Virginia Polytechnic Institute and State University; 2004.

[22] C.-S. Park, D. Avirovik, S. Bressers, S. Priya, Low-frequency nanotesla sensitivity in Metglas/piezoelectric/carbon fiber/piezoelectric composites with active tip mass, Applied Physics Letters, 98(2011) 1-3.

[23] Y. Zhou, D.J. Apo, S. Priya, Dual-phase self-biased magnetoelectric energy harvester, Applied Physics Letters, 103(2013) 192909. 


\section{Figure(if any)}

a)
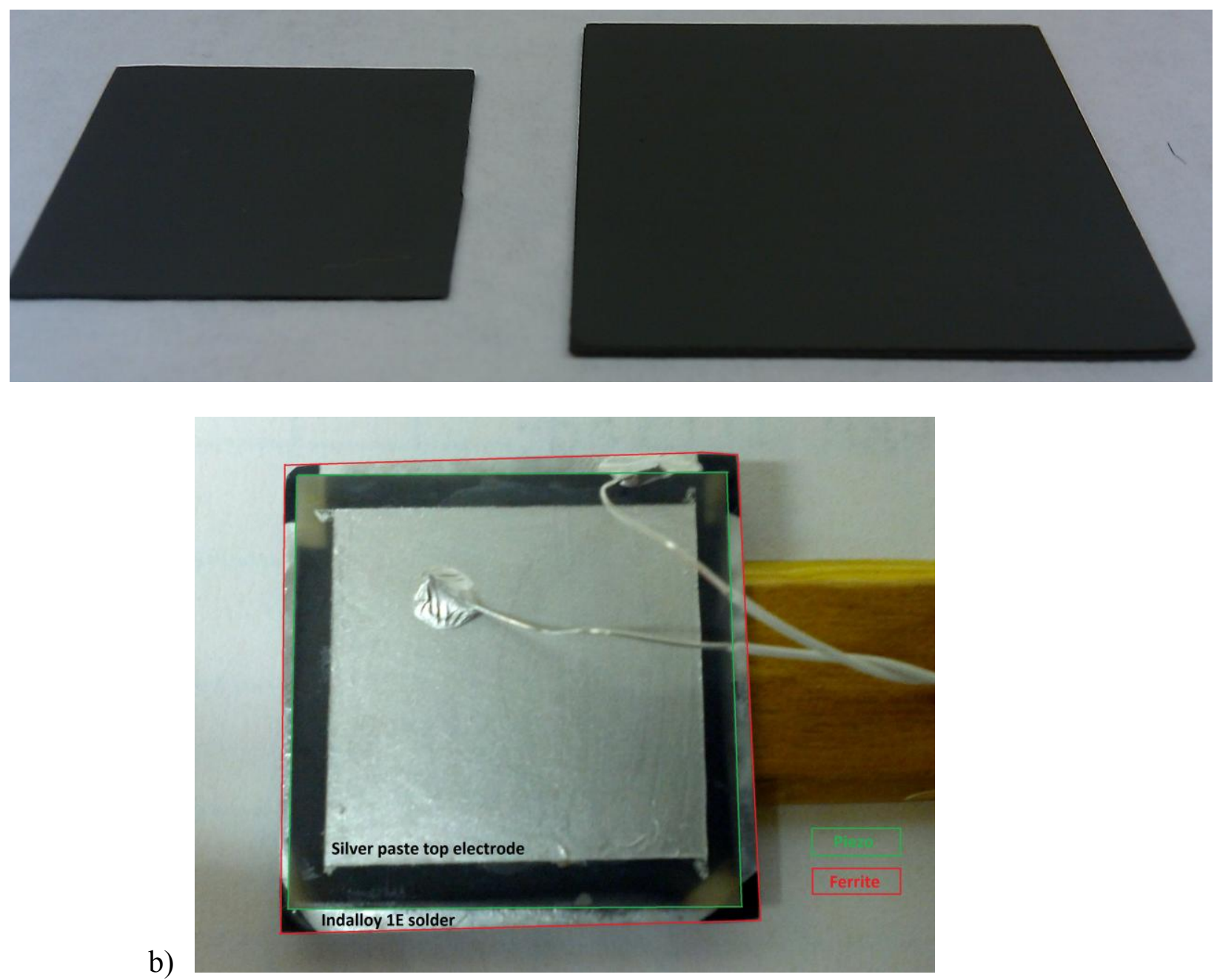

c)

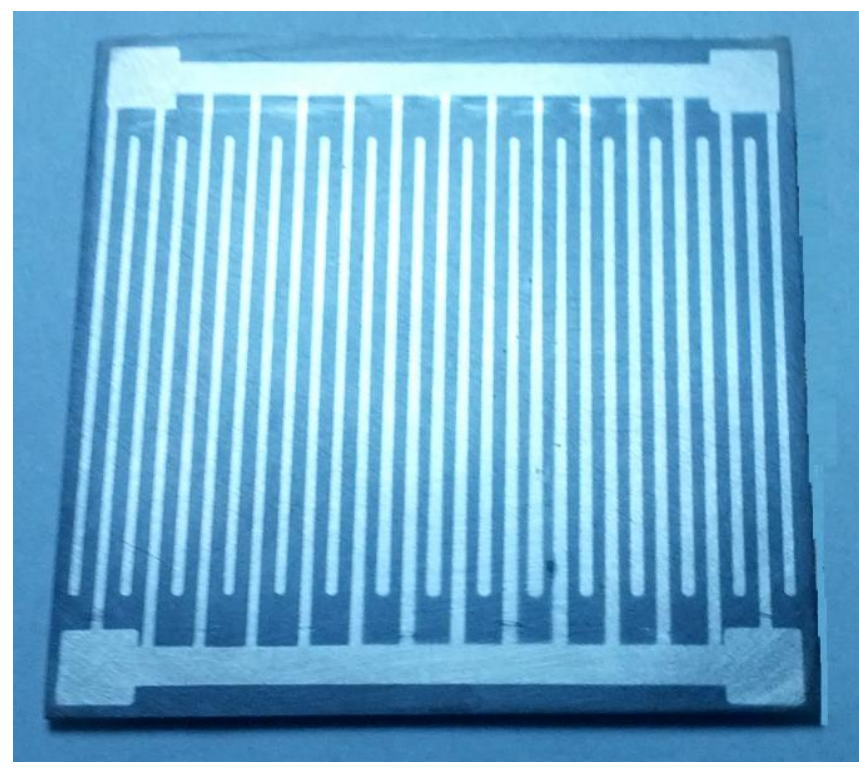


Figure 1 a) Ferrite 40011 fired sheet and ME soldered composite with b) $d_{31}$ mode electroding using silver paste conductors and c) $d_{33}$ mode electroding using Pt conductors.
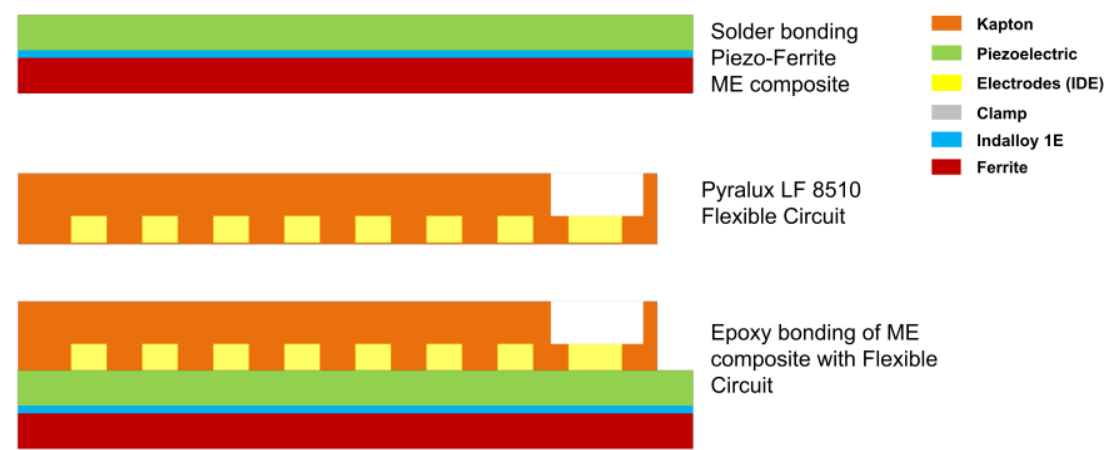

Epoxy bonding of ME

composite with Flexible

Circuit

Figure 2 Schematic of the fabrication process flow for ME macro fiber composite cantilever

a)

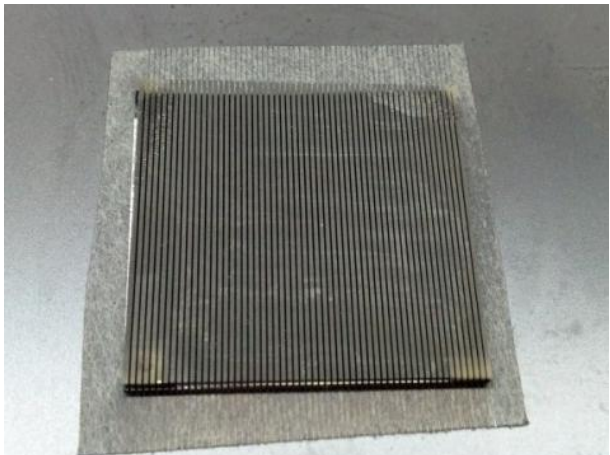

b)

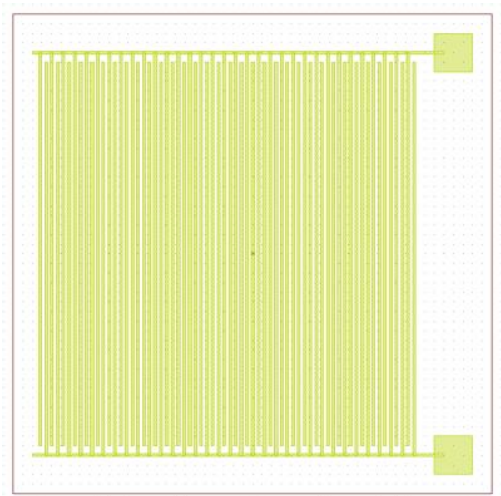




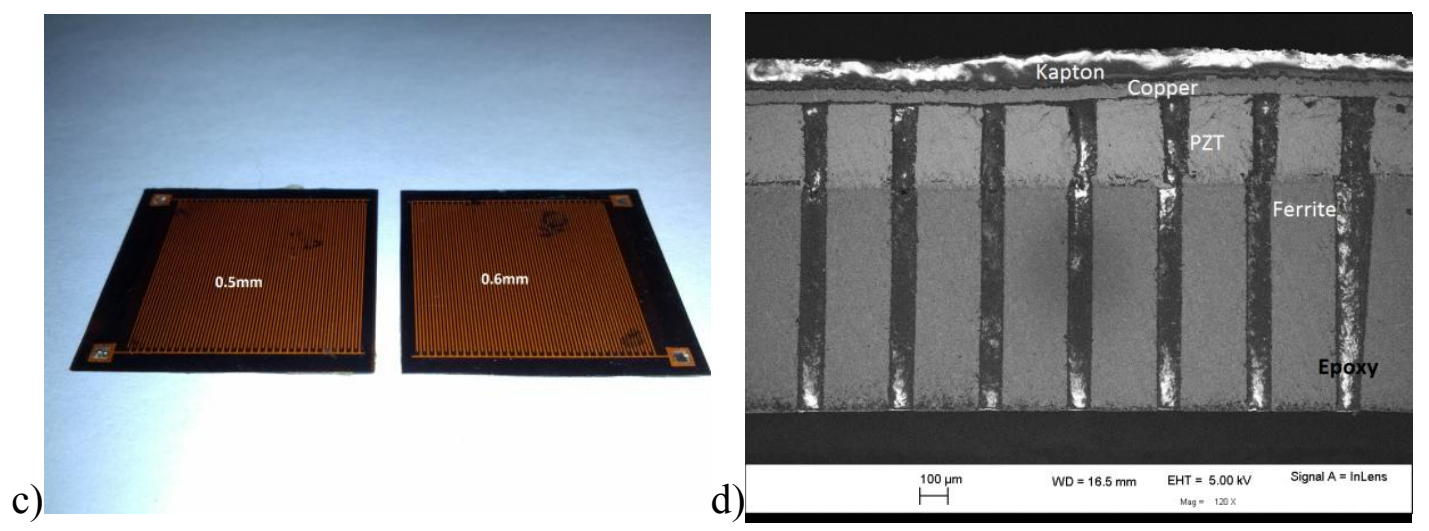

Figure 3 a) diced ME composite b) IDE pattern for flexible circuit and c) final ME macro fiber composite (ferrite of $0.5 \mathrm{~mm}$ on left and $0.6 \mathrm{~mm}$ on right) and d) SEM cross section of the ME MFC.

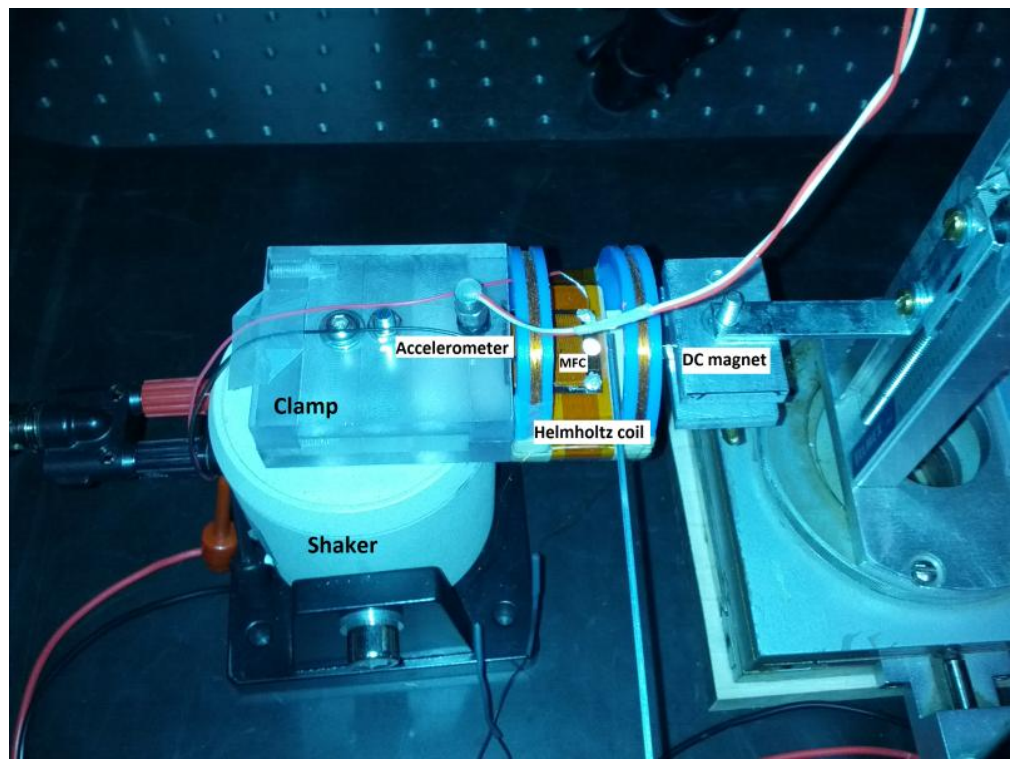

Figure 4 Magnetoelectric test setup with a translatable DC magnetic bias shown on the right side. 


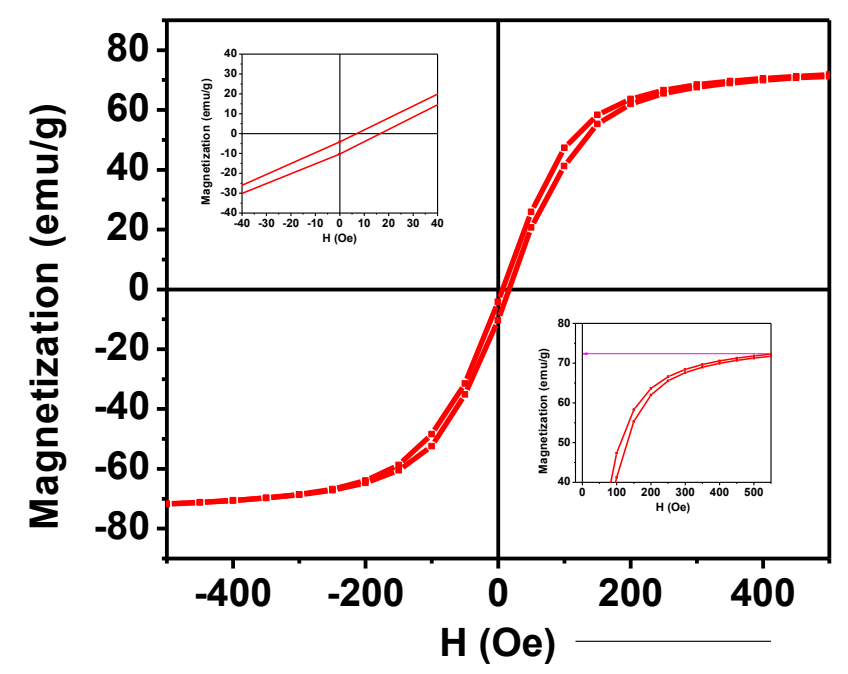

a)

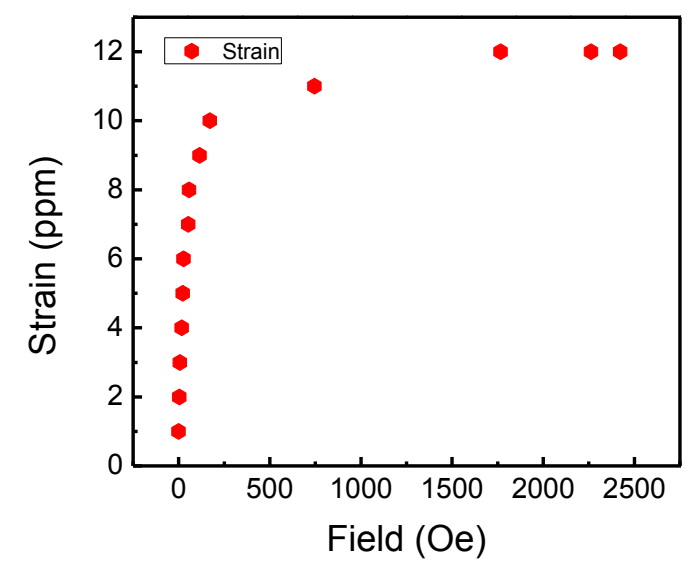

Figure 5 a) Magnetization-Field (M-H) hysteresis loop and b) Magnetostriction results for Electroscience Type 40011 ferrite 


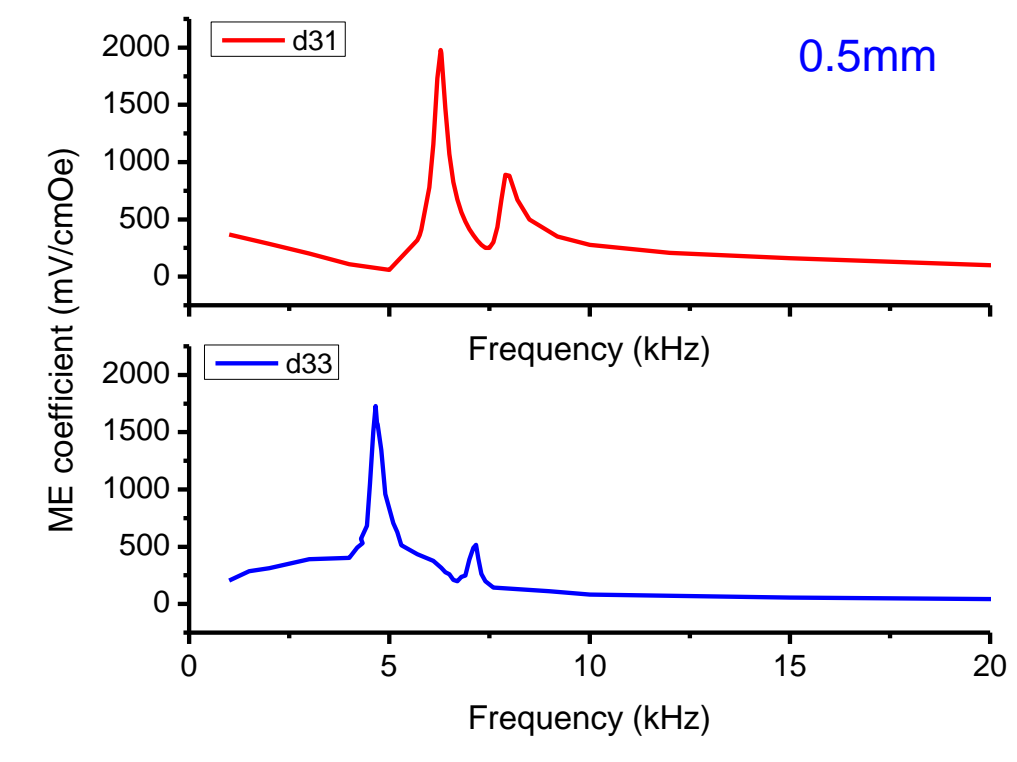

a)

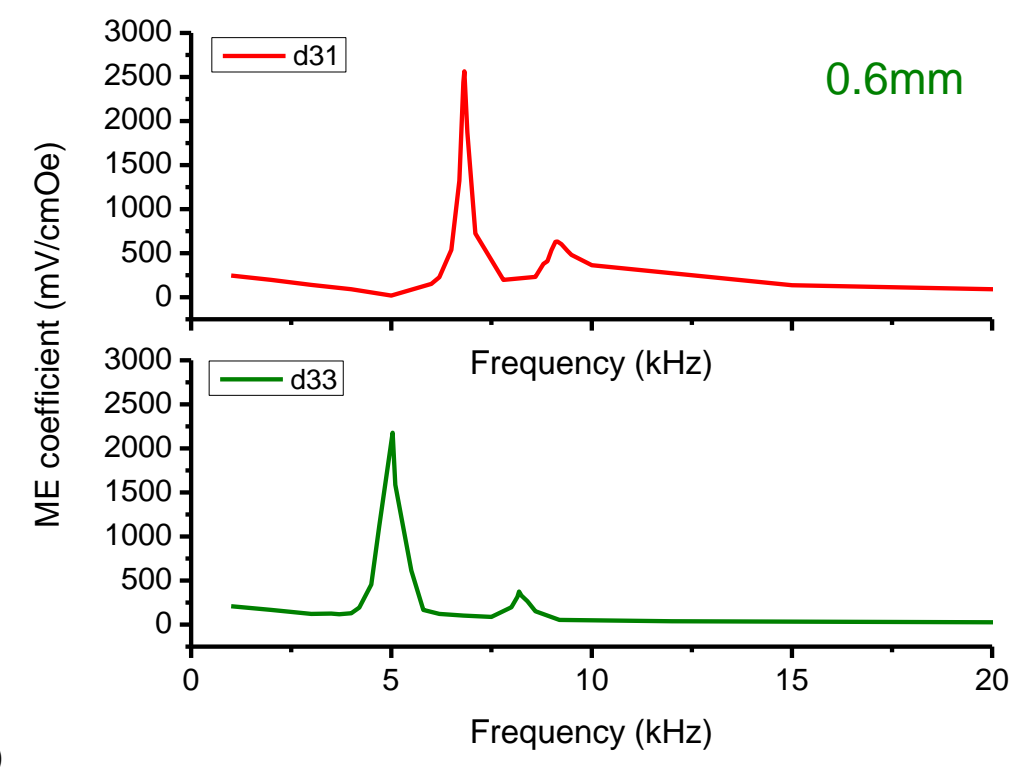

Figure 6 Magnetoelectric voltage coefficient results for a) $0.5 \mathrm{~mm}$ Ferrite and b) $0.6 \mathrm{~mm}$ Ferrite ME composites operating in $\mathrm{d} 31$ and d33 modes. 


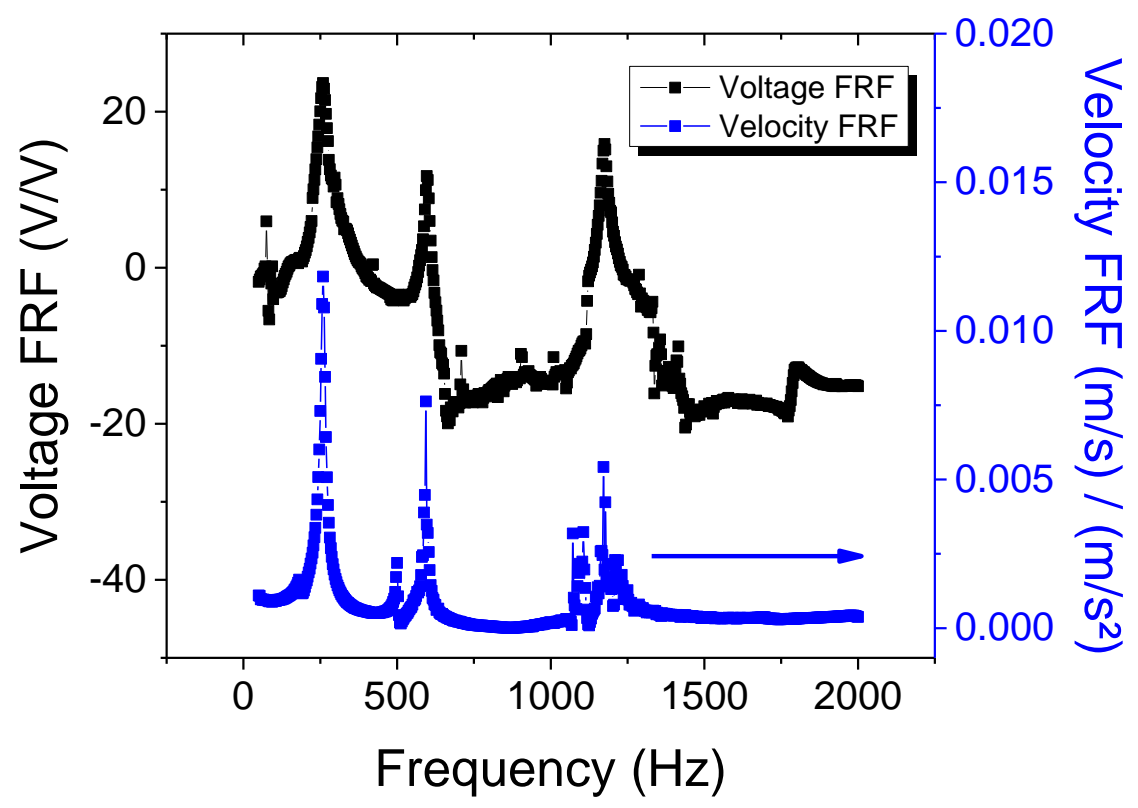

Figure 7 Voltage (top) and Velocity frequency response functions for the $0.5 \mathrm{~mm}$ Ferrite MFC 


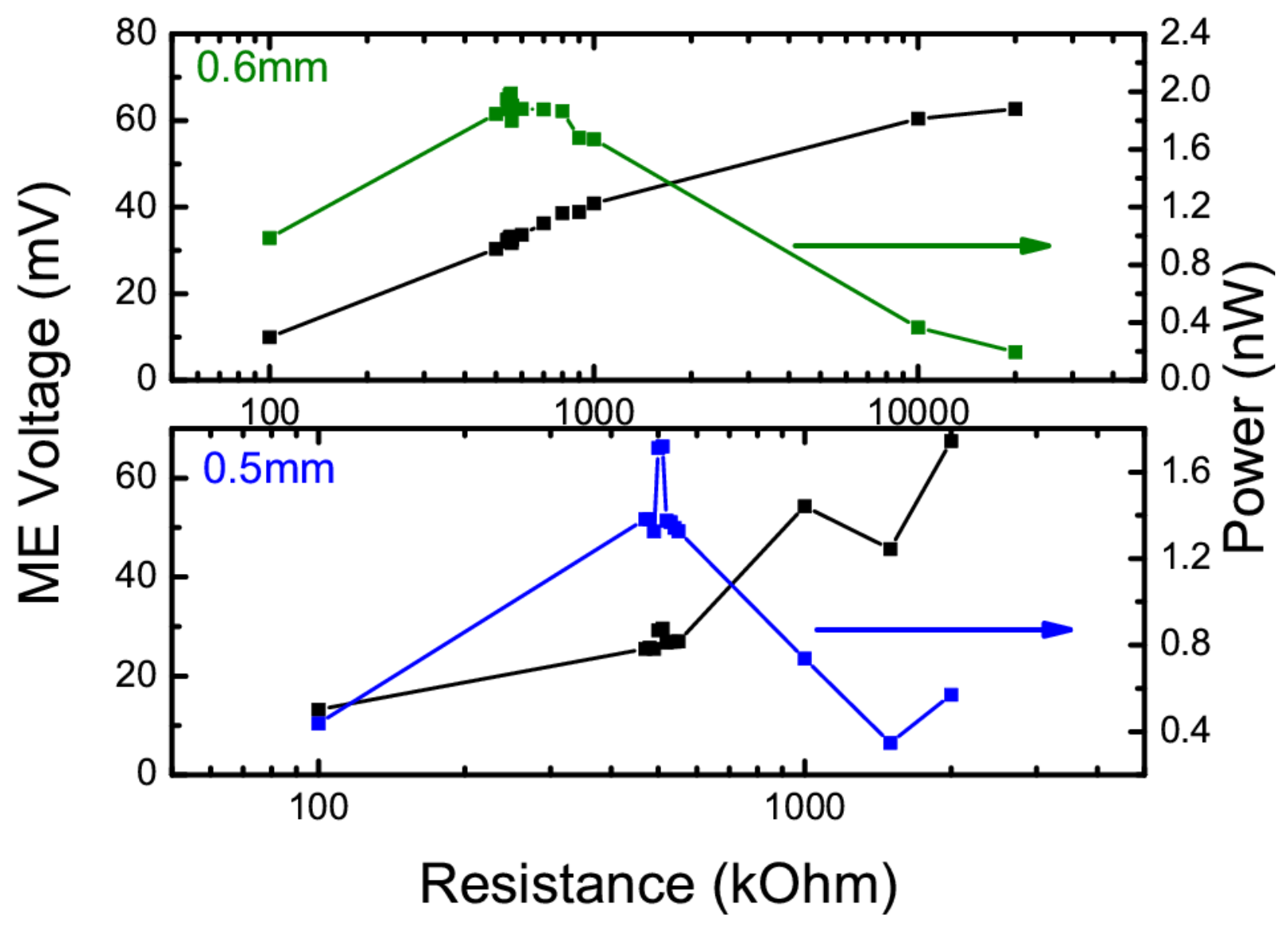

Figure 8 Voltage and power loading curves for $0.6 \mathrm{~mm}$ (top) and $0.5 \mathrm{~mm}$ (bottom) ME composite MFC's. 
Table 1: Summary of the ME voltage results for ME composites vs. Ferrite thickness

\begin{tabular}{lccccccc}
\hline & \multicolumn{8}{c}{ Ferrite Thickness(mm) } \\
\hline & \multicolumn{6}{c}{$\mathbf{0 . 5}$} & \multicolumn{4}{c}{$\mathbf{0 . 6}$} \\
& mode & $\mathbf{1}^{\text {st }}$ & $\mathbf{2}^{\text {nd }}$ & Ratio & $\mathbf{1}^{\text {st }}$ & $\mathbf{2}^{\text {nd }}$ & Ratio \\
\hline Frequency response (kHz) & $\mathrm{d} 31$ & 6.31 & 7.98 & 1.26 & 6.82 & 9.15 & 1.34 \\
& $\mathrm{~d} 33$ & 4.66 & 7.15 & 1.53 & 5.04 & 8.19 & 1.63 \\
ME coeff. (mV/cmOe) & $\mathrm{d} 31$ & 1985 & 893 & & 2574 & 634 & \\
& $\mathrm{~d} 33$ & 1730 & 515 & & 2177 & 376 & \\
\hline
\end{tabular}

Table 2: MFC harvesting and sensing performance

\begin{tabular}{llllllll}
\hline Ferrite & AC & AC & DC & $\mathbf{V}_{\text {OC }}$ & $\mathbf{R}_{\text {load }}$ & Power & Sensitivity \\
thickness & $(\mathrm{Oe})$ & $(\mathrm{Hz})$ & $(\mathrm{Oe})$ & $(\mathrm{mV})$ & $(\mathrm{k} \Omega)$ & $(\mathrm{nW})$ & $(\mathrm{mV} / \mathrm{mT})$ \\
\hline $0.6 \mathrm{~mm}$ & 1 & 264 & 22 & 101.1 & 356 & 3.1 & 1011 \\
$0.5 \mathrm{~mm}$ & 1 & 259 & 28 & 93.84 & 337 & 2.6 & 938 \\
\hline
\end{tabular}




\title{
Ronnie Varghese, ${ }^{1}$ Shree Narayanan, ${ }^{3}$ Donald Leber, ${ }^{3}$ Ravindranath Viswan, ${ }^{2}$ Mingkai $\mathrm{Mu},{ }^{3}$ Mohan
} Sanghadasa $^{4}$ and Shashank Priya ${ }^{1}$

\author{
${ }^{I}$ Center for Energy Harvesting Materials and Systems (CEHMS), Bio-Inspired Materials and Devices Laboratory (BMDL), Virginia Tech, \\ Blacksburg, VA 24061, USA \\ ${ }^{2}$ Materials Science and Engineering Department, Virginia Tech, Blacksburg, VA 24061, USA \\ ${ }^{3}$ Electrical and Computer Engineering Department, Virginia Tech, Blacksburg, VA 24061, USA \\ ${ }^{4}$ Weapons Sciences Directorate, U.S. Army Research, Development, and Engineering Command, Redstone Arsenal, AL 35898
}

